Adsorbate-induced one-dimensional long-range modulation of an epitaxial insulator film

W. Ernst, M. Eichmann, H. Pfnür, K.-L. Jonas, V. von Oeynhausen, and K. H. Meiwes-Broer

Citation: Appl. Phys. Lett. 80, 2595 (2002);

View online: https://doi.org/10.1063/1.1467624

View Table of Contents: http://aip.scitation.org/toc/apl/80/14

Published by the American Institute of Physics

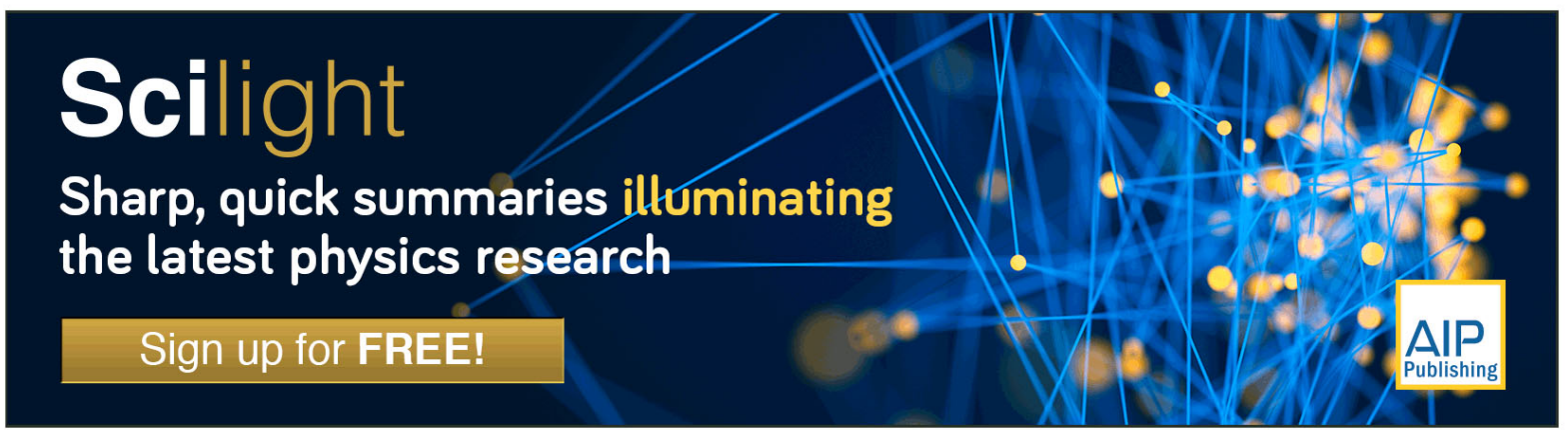




\title{
Adsorbate-induced one-dimensional long-range modulation of an epitaxial insulator film
}

\author{
W. Ernst, M. Eichmann, and H. Pfnür ${ }^{\mathrm{a})}$ \\ Institut für Festkörperphysik, Universität Hannover, Appelstraße 2, D-30167 Hannover, Germany \\ K.-L. Jonas, V. von Oeynhausen, and K. H. Meiwes-Broer \\ Fachbereich Physik, Universität Rostock, Universitätsplatz, 3, D-18055 Rostock, Germany
}

(Received 18 December 2001; accepted for publication 13 February 2002)

\begin{abstract}
Using low-energy electron diffraction and scanning tunneling micrsocopy, we found that epitaxial $\mathrm{NaCl}$ films grown on $\mathrm{Ge}(100)$ with thicknesses up to (at least) 15 monolayers can be modulated with a period of six lattice constants and an amplitude directed mainly normal to their surface. The (6 $\times 1)$ periodicity on the $\mathrm{NaCl}$ films is induced by a preadsorbed $\mathrm{Na}$ layer at very low coverages $(\Theta \approx 0.06)$, that form chain structures with a sixfold periodicity in one dimension. At 10 monolayers thickness of $\mathrm{NaCl}$ a modulation amplitude of $0.28 \AA$ was obtained. () 2002 American Institute of Physics. [DOI: 10.1063/1.1467624]
\end{abstract}

Very thin insulating films have a wide range of technical applications from chemistry and catalysis to electronics. Especially the miniaturization of electronic devices requires further reductions of size and a precise definition of interfaces on the atomic scale, which can be best controlled by epitaxial or even pseudomorphic growth. There is also a need for structuring of materials on these small scales, which can be greatly facilitated by a prestructuring due to selforganization, especially at the conductor/insulator interfaces. Thus physical properties of extremely small systems as well as perspectives of their applicability could be tested. An example of prestructuring of an epitaxial insulating film by strain modulation on the nanometer scale is reported here. This modulation opens the possibility of preferential deposition of other materials in the strained sections of the insulator and to create patterns of conducting material on an insulator.

The main problem of the insulator/conductor interface is the large difference in surface free energies of an insulator and a metal, which in many cases prevents wetting of a metallic layer on an insulator. For the same reason, perfect surfaces of a wide class of insulators are chemically rather inert. ${ }^{1-3}$ This chemical inertness is necessarily also coupled with weak interactions of an insulating film with its substrate, which contrasts with the comparatively high cohesive energies witin the insulator. This is the reason, why epitaxial growth of these films requires generally a close match between lattice constants of insulator and substrate. ${ }^{4,5}$ For this reason it is generally difficult to force an insulating film to follow the structure of its support, but the opposite, structuring of the substrate by an insulating film, has been reported. ${ }^{6}$

The $\mathrm{NaCl} / \mathrm{Ge}(100)$ system seems to be a good test candidate to explore many possiblities for structuring of insulating layers. Not only does the close match of lattice constants allow almost perfect epitaxial growth, ${ }^{7}$ steps of monatomic height of $\mathrm{Ge}$ that do not fit to the $\mathrm{NaCl}$ lattice are overgrown elastically, ${ }^{5}$ i.e., the $\mathrm{NaCl}$ films are not rigid on a mesoscopic scale. These films turned out to be free of defects for meth-

${ }^{a)}$ Electronic mail: pfnuer@fkp.uni-hannover.de ods like electron spectroscopy and adsorption experiments. Double steps of Ge in [1 $1 \overline{1} 0]$ direction, on the other hand, seem to be able to force the formation of steps in this same direction also on the surface of a thin $\mathrm{NaCl}$ layer, in which the steps end by a single sort of ions, i.e., they are polar. ${ }^{8}$ Surface color centers, i.e., point-like anion vacancies, offer the possibility of structuring by binding adsorbed atoms and molecules more strongly. This is why their properties have been investigated in detail on $\mathrm{NaCl} .{ }^{9-11}$

The possibility of periodic modulations of $\mathrm{NaCl}$ films on $\mathrm{Ge}(100)$ on an almost atomic scale is demonstrated here. As will be shown, it is due to a combination of geometric height modulation and a modulated binding strength to the substrate, both induced by small concentrations of preadsorbed and annealed Na. Since the geometric structure of the $\mathrm{Na}$ adsorbate is crucial for the modulation of the $\mathrm{NaCl}$ films, we first describe this structure obtained with scanning tunneling microscopy (STM) and low-energy electron diffraction (LEED), before we turn to the quantitative LEED analysis of a modulated $\mathrm{NaCl}$ film. LEED experiments on the $\mathrm{NaCl}$ films have been carried out in a $\mu$-metal shielded UHV chamber at a base pressure of $5 \times 10^{-9} \mathrm{~Pa}$. For the STM experiments a system with low temperature STM and a preparation chamber was used. ${ }^{12}$ Sample treatment and sample preparation of the samples used in both systems were identical. In UHV, sputter/annealing cycles have been carried out until no contamination was detectable with Auger electron spectroscopy, and LEED revealed a brilliant $(2 \times 1)$ structure. Average terrace widths of $500 \AA$ are typical for these samples, as checked with LEED and STM. Temperatures around $80 \mathrm{~K}$ have been reached by cooling the sample with $\mathrm{LN}_{2}$ using a flow cryostat. The samples have been heated either radiatively or by electron bombardment. Details of the setup are described in Ref. $11 . \mathrm{NaCl}$ was evaporated as molecules from an $\mathrm{Al}_{2} \mathrm{O}_{3}$ tube filled with $\mathrm{NaCl}$ powder (purity 99.9995\%). Typical rates of evaporation of 0.2 $\mathrm{ML} / \mathrm{min}$ have been controlled by a quartz microbalance.

Dissociation experiments on $\mathrm{NaCl}$ films with electron beams showed clearly that residual $\mathrm{Na}$ remaining on the bare Ge surface was the reason for the modulation of the $\mathrm{NaCl}$ 


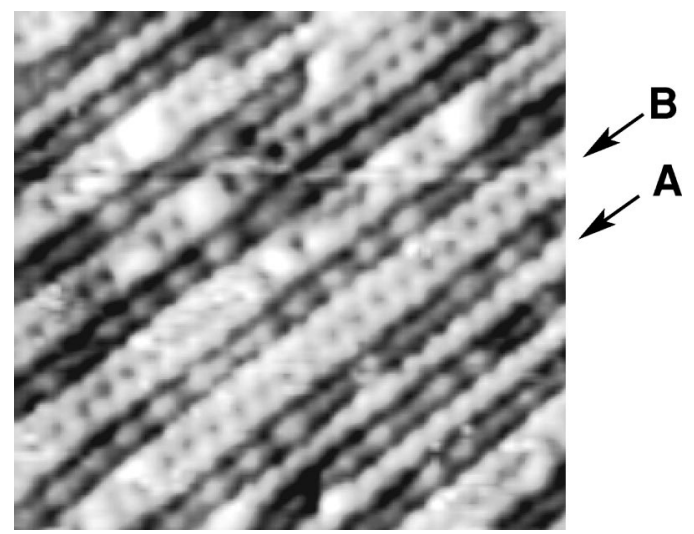

FIG. 1. STM image of the low coverage $(6 \times 1)$ structure induced by adsorbed $\mathrm{Na}$ on $\mathrm{Ge}(100)$. Surface temperature $80 \mathrm{~K}$. Single and double dimer rows of $\mathrm{Ge}$ are marked with $\mathrm{A}$ and $\mathrm{B}$, respectively.

films subsequently adsorbed. In order to provide direct evidence and to determine the amount and structure of the $\mathrm{Na}$ layer on $\mathrm{Ge}(100)$ necessary to induce the observed modulation of the $\mathrm{NaCl}$ layer adsorbed on top, we evaporated $\mathrm{Na}$ onto the clean $\mathrm{Ge}(100)$ surface and carried out LEED and STM measurements on layers of various coverages without braking the vacuum. Starting at a coverage of $0.5 \mathrm{ML}$, coverages were successively reduced to zero by partial desorption at temperatures between 650 and $750 \mathrm{~K}$. The original $(4 \times 1)$ structure, assumed to correspond to half a monolayer of $\mathrm{Na}^{13}$ was transformed into a $(3 \times 2)$ structure, and upon further desorption into a $(6 \times 1)$ LEED pattern with streaks. Only this structure turned out to be able to induce periodic modulations of $\mathrm{NaCl}$ layers adsorbed on top of this structure in a wide range of layer thicknesses of $\mathrm{NaCl}$.

More detailed geometrical information about this $\mathrm{Na}$ layer was obtained from STM. In Fig. 1 we show a STM image in constant current mode for tunneling conditions from filled states of the Ge surface with atomic resolution. The bright rows can easily identified as single and double rows of Ge dimers, since they show the typical bias dependence of contrast that was also found on the bare Ge surface for the Ge dimers. They are arranged in $(2 \times 1)$ periodicity at room temperature and in a $c(4 \times 2)$ structure at $80 \mathrm{~K}$ used in our STM experiments. The dark rows can be identified as $\mathrm{Na}$ rows with a separation of three lattice constants along each row. At the location of the $\mathrm{Na}$ rows the Ge dimer rows are absent. At the coverage shown the double Ge dimer rows appear with high preference. Together with the Na rows they form the six-fold peridicity observed in LEED. The superperiodicity in the Na rows turns out to be uncorrelated between

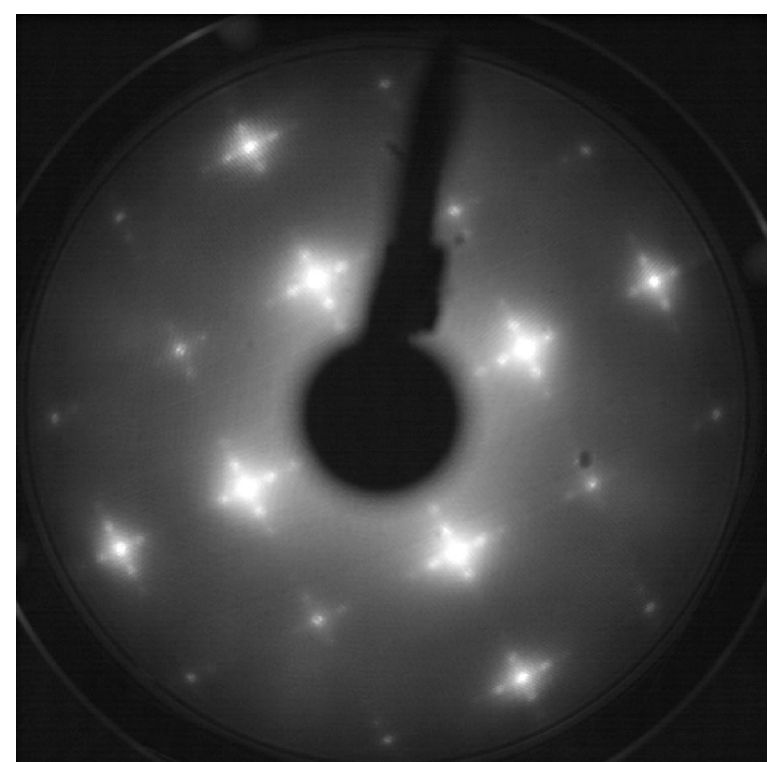

FIG. 2. LEED pattern of a 10 -ML-thick $\mathrm{NaCl}$ film. As clearly seen, the $(1 \times 1)$ spots have satellite spots of the $(6 \times 1)$ modulation.

different rows. As additional experiments revealed, the $\mathrm{Na}$ coverage determines the probability for single and double $\mathrm{Ge}$ dimer rows to occur, which can be continuously adjusted by the Na concentration. The Na coverage for a pure $(6 \times 1)$ structure corresponds to $0.055 \mathrm{ML}$.

When more than $2 \mathrm{ML}$ of $\mathrm{NaCl}$ are adsorbed onto a $\mathrm{Ge}(100)$ surface precovered with the concentration of Na just shown, all LEED spots show a structure with characteristic satellites (see Fig. 2) instead of the $(1 \times 1)$ structure seen for $\mathrm{NaCl}$ on the clean $\mathrm{Ge}(100)$ surface. These satellites do not change position in $k_{\|}$when the electron energy in LEED is changed, indicating that no mosaics or facets are formed, i.e., the structure is modulated. The distance between the satellites corresponds to exactly the six lattice constants of the $\mathrm{Na}$ modified Ge surface already found with STM, and again two domains are formed.

From the increase of the satellite intensities relative to those of the central integer order spots as a function of electron energy (see Fig. 3) we conclude ${ }^{14}$ that the periodic relaxations are mainly in the direction normal to the surface. For a quantitative analysis, we integrated the center and a ring containing the first order satellites, as shown schematically on the right side of Fig. 3.

Since multiple electron scattering is limited to a few lattice constants, this fairly long range modulation (six lattice constants) can be analyzed within the kinematic scattering

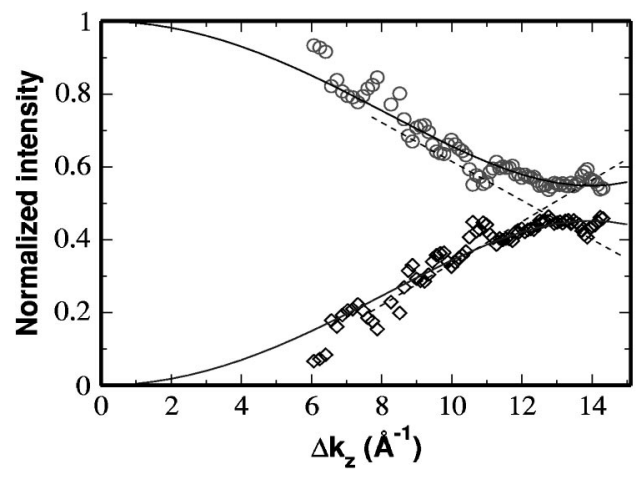

Integration of data:

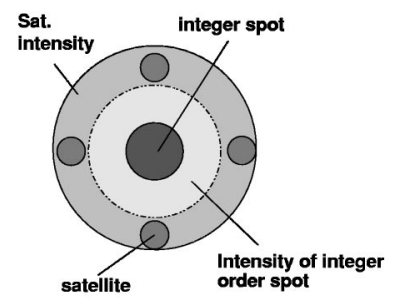

FIG. 3. Left: Relative intensities of satellites $(\diamond)$ and integer order beams $(\bigcirc)$ as a function of electron energy. Right: Enlarged sketch of a spot with satellites is shown. The differently shaded areas mark areas of integration integer order spots and satellites. 
approximation. The smooth dependence of the relative intensities shown in Fig. 3 on electron energy corroborates this assumption. The LEED pattern indicates that the modulation is one-dimensional on each terrace. We therefore used a simple one-dimensional kinematic model to simulate the experimentally obtained curves of Fig. 3. Here it was assumed that the $\mathrm{Na}^{+}$and $\mathrm{Cl}^{-}$ions are positioned laterally on regular lattice sites, whereas the positions normal to the surface are modulated by a height function

$$
h(z)=A_{1} \cos \frac{2 \pi x}{6 a_{0}}+A_{2} \cos \frac{2 \pi x}{6 a_{0}}+\ldots
$$

We found close resemblance of the simulated energy dependencies of relative intensities with those experimentally determined. This justifies our approach and also indicates that the main contribution to the modulation is in fact normal to the surface.

A quantitative analysis of experimental data has been carried out for a 10-ML-thick $\mathrm{NaCl}$ film. For this thickness we can be sure that the electrons in the LEED experiment only see the $\mathrm{NaCl}$ film, not the interface, i.e., structural properties of the interface such as reconstructions and relaxations do not contribute to the LEED intensity. The best fit to the experimental data using just the first Fourier component is indicated in Fig. 3 as dashed lines. They show systematic deviations from the experimental data points at high energies. However, the fit describes the data satisfactorily already when a second Fourier component was included (see full line in Fig. 3). Best fits have been obtained with $A_{1}=0.095 \AA$ and $A_{2}=0.090 \AA$. Thus, the maximum height variation is $0.28 \AA$ for the 10 -ML-thick film.

This characteristic two-peak modulation can easily be rationalized from the STM images of the Na modulated surface (Fig. 1), if we identify the maxima in the height modulation function with the positions of the Ge dimer rows. The valleys in this modulation must then correspond to the position of the Na rows. A schematic model of this modulational structure is shown in Fig. 4. This assignment seems to be plausible for two reasons. First, from $\mathrm{x}$-ray scattering data it is known that the dimer rows of Ge survive after adsorption of $\mathrm{NaCl}$, whereas the $c(4 \times 2)$ reconstruction disappears. ${ }^{15} \mathrm{In}$ addition, for very thin layers $(<5 \mathrm{ML})$ the reappearance of a $(2 \times 1)$ LEED pattern is reported once the first double layer of $\mathrm{NaCl}$ is completed. ${ }^{10}$ This means that even on the flat surface the $\mathrm{NaCl}$ films are slightly modulated by the dimer row structure of the $\mathrm{Ge}$ interface. It explains why for a double dimer row structure at the interface an additional minimum of a Na modified surface due to additional relaxations of the Ge dimers. Second, the pronounced minimum at the position of the Na rows indicates that binding of these $\mathrm{Na}$ rows, presumably to the $\mathrm{Cl}$ ions of the $\mathrm{NaCl}$ layer, is stronger

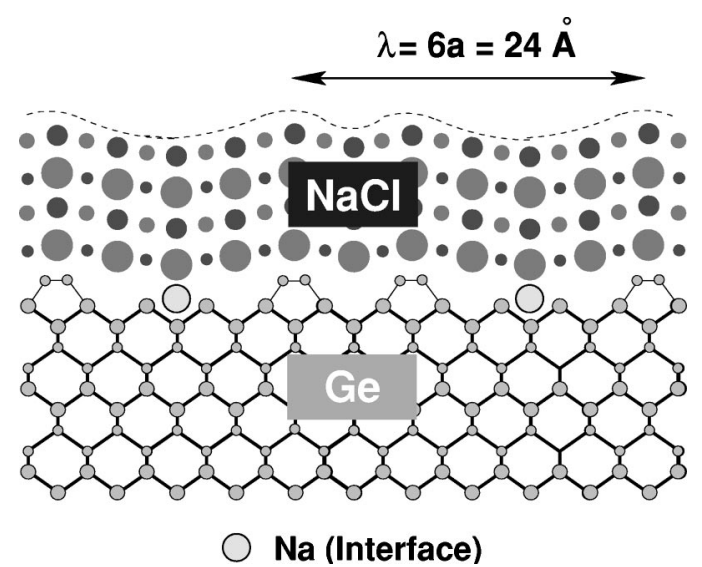

FIG. 4. Proposed schematic geometrical model of the $\mathrm{Na}$ induced height modulation of a $\mathrm{NaCl}$ film. Dashed line: Height modulation obtained from fit to data of Fig. 3 .

than between $\mathrm{NaCl}$ and $\mathrm{Ge}$. The modified chemical properties and modifications of the geometrical environment at the positions of the Na rows compared with the dimer configuration might help to deepen the valley seen there.

Summarizing, this study shows that the interaction between semiconductor and insulator can be enhanced sufficiently by an ordered array of atoms adsorbed at the interface so that regular modulations of the insulator film are obtained. This opens a variety of possibilities for self-organized structures on insulating films on the nanometer scale.

The authors thank Dr. Ch. Tegenkamp for many inspiring discussions and the K\&S AG for financial support.

${ }^{1} \mathrm{~V}$. Henrich and P. Cox, The surface science of metal oxides (Cambridge University Press, Cambridge, 1996).

${ }^{2}$ C. Henry, Surf. Sci. Rep. 31, 231 (1998).

${ }^{3}$ M. Bäumler and H. J. Freund, Prog. Surf. Sci. 61, 127 (1999).

${ }^{4}$ X. Lai, Q. Guo, B. K. Bin, and D. W. Goodman, Surf. Sci. 487, 1 (2001).

${ }^{5}$ C. Schwennicke, J. Schimmelpfennig, and H. Pfnür, Surf. Sci. 293, 57 (1993).

${ }^{6}$ J. Repp, S. Fölsch, G. Meyer, and K. H. Rieder, Phys. Rev. Lett. 86, 252 (2001).

${ }^{7}$ S. Fölsch, U. Barjenbruch, and M. Henzler, Thin Solid Films 127, 123 (1989).

${ }^{8}$ C. Tegenkamp, W. Ernst, and H. Pfnür, Surf. Sci. 466, 41 (2000).

${ }^{9}$ U. Malaske, C. Tegenkamp, H. Pfnür, and M. Henzler, Surf. Sci. 408, 237 (1998).

${ }^{10}$ V. Zielasek, T. Hildebrandt, and M. Henzler, Phys. Rev. B 62, 2912 (2000).

${ }^{11}$ W. Ernst, M. Eichmann, K. Budde, and H. Pfnür, Surf. Sci. 464, 35 (2000).

${ }^{12}$ A. Bettac, L. Koller, V. Rank, and K. H. Meiwes-Broer, Surf. Sci. 402404, 475 (1998)

${ }^{13}$ B. Naydenov and L. Surnev, Surf. Sci. 370, 155 (1997).

${ }^{14}$ M. Horn-von Hoegen, Z. Kristallogr. 214, 1 (1999).

${ }^{15}$ C. A. Lucas, G. C. L. Wong, C. S. Dower, F. J. Lamelas, and P. H. Fuoss, Surf. Sci. 286, 46 (1993). 